\title{
CDISC CDASH Unit of Measure for Flow Rate Terminology
}

National Cancer Institute

\section{Source}

National Cancer Institute. CDISC CDASH Unit of Measure for Flow Rate Terminology. NCI Thesaurus. Code C78429.

A terminology subset of the CDISC Study Data Tabulation Model (SDT M) Unit codelist created for Clinical Data Acquisition Standards Harmonization (CDASH) Unit of Measure for Flow Rate terminology. 